\title{
Perfil epidemiológico da violência contra a pessoa idosa
}

\author{
Epidemiological profile of violence against the elderly \\ Perfil epidemiológico de la violencia contra las personas mayores
}

Recebido: 15/01/2022 | Revisado: 19/01/2022 | Aceito: 25/01/2022 | Publicado: 26/01/2022

Daniele Duarte do Nascimento

ORCID: https://orcid.org/0000-0003-0542-3734

Universidade Federal de Alagoas, Brasil

E-mail: duarte1328@hotmail.com

Elizabeth Moura Soares de Souza

ORCID: https://orcid.org/0000-0003-0727-2677

Universidade Federal de Alagoas, Brasil E-mail: elmososo@gmail.com

Rebeca Thomé Costa Santa Cruz ORCID: https://orcid.org/0000-0003-0727-2677

Universidade Estadual de Alagoas, Brasil E-mail: rebeca-tc@hotmail.com

Thaís Honório Lins Bernardo

ORCID: https://orcid.org/0000-0002-8058-8400

Universidade Federal de Alagoas, Brasil

E-mail: thais.bernardo@esenfar.ufal.br

\begin{abstract}
Resumo
Objetivo: Descrever o perfil epidemiológico da violência contra a pessoa idosa de um estado da região nordeste do Brasil. Metodologia: Estudo quantitativo, descritivo, retrospectivo, realizado com informações disponíveis em um banco de dados do Sistema de Informação de Agravos e Notificação, no período de 2008 a 2018. A análise dos dados ocorreu através da estatística simples (frequência e percentual). Resultados: Foram analisadas 1.535 fichas de notificação de violência, sendo que houve um número grande de campos ignorados e em branco. Em relação às características das pessoas idosas, $63,4 \%$ eram homens, 34,8\% de raça parda, 23,8\% tinham um nível de escolaridade entre analfabeto e ensino fundamental, 71,9\% vítimas sofreram violência física, sendo $41,3 \%$ através da força corporal/espancamento, $56,8 \%$ dos casos ocorreram na zona urbana, $37,9 \%$ na própria residência da vítima, $74,4 \%$ classificada como interpessoal e $37,2 \%$ foram notificadas por unidades de emergência. Conclusão: A violência ocorrida contra as pessoas idosas ocorreu em sua maioria nos homens, alfabetizados através de foça corporal/espancamento. Este estudo evidencia que a incompletude de informações no preenchimento da ficha de notificação revela a necessidade de um olhar ampliado da equipe de saúde para o registro completo dos dados.
\end{abstract}

Palavras-chave: Idoso; Violência; Enfermagem; Epidemiologia; Envelhecimento.

\begin{abstract}
Objective: To describe the epidemiological profile of violence against the elderly in a state in the northeast region of Brazil. Methodology: Quantitative, descriptive, retrospective study, carried out with information available in a database of the Diseases Information System and Notification, from 2008 to 2018. Data analysis was performed using simple statistics (frequency and percentage). Results: 1,535 violence notification forms were analyzed, with a large number of ignored and blank notifications. Regarding the characteristics of the elderly, $63.4 \%$ were men, $34.8 \%$ of mixed race, $23.8 \%$ with an education level between illiterate and elementary school, $71.9 \%$ victims of physical violence, $41,3 \%$ through physical force/beating, $56.8 \%$ of the violence occurred in the urban area, $37.9 \%$ in the victim's own residence, $74.4 \%$ classified as interpersonal and $37.2 \%$ were notified by emergency units. Conclusion: Violence against elderly people occurred mainly in men, who were literate and who were victims of physical force/beating. This study shows that the incompleteness of information in filling out the notification form reveals the need for a broader view of the health team for the complete recording of data.
\end{abstract}

Keywords: Violence; Nursing; Epidemiology; Aging.

\section{Resumen}

Objetivo: Describir el perfil epidemiológico de la violencia contra el adulto mayor en un estado de la región noreste de Brasil. Metodología: Estudio cuantitativo, descriptivo, retrospectivo, realizado con información disponible en una base de datos del Sistema de Información y Notificación de Enfermedades, de 2008 a 2018. El análisis de los datos se realizó mediante estadística simple (frecuencia y porcentaje). Resultados: se analizaron 1.535 formularios de notificación de violencia, con una gran cantidad de notificaciones ignoradas y en blanco. En cuanto a las características de los adultos mayores, $63,4 \%$ eran hombres, $34,8 \%$ mestizos, $23,8 \%$ con nivel educativo entre 
analfabetos y primaria, 71,9\% víctimas de violencia física, 41,3\% por fuerza física / golpes, 56,8\% de la violencia ocurrió en el área urbana, el 37,9\% en la propia residencia de la víctima, el 74,4\% clasificó como interpersonal y el $37,2 \%$ fueron notificados por las unidades de emergencia. Conclusión: La violencia contra las personas mayores ocurrió principalmente en hombres, que sabían leer y escribir y que fueron víctimas de fuerza física / golpiza. Este estudio muestra que la falta de información al completar el formulario de notificación revela la necesidad de una visión más amplia del equipo de salud para el registro completo de los datos.

Palabras clave: Anciano; Violencia; Enfermería; Epidemiología; Envejecimiento.

\section{Introdução}

O Brasil vem vivenciando mudanças estruturais em sua pirâmide etária marcada pelo acelerado envelhecimento populacional e percebe-se que a sociedade não está acompanhando esta transição demográfica, deixando a desejar no trato com os idosos, por isso se faz necessário dispor de redes de serviço capacitados e pessoas preparadas para lidar com os idosos na prevenção da qualidade de vida na terceira idade (Paraíba \& Silva 2015).

A Organização Mundial da Saúde (OMS) define a violência contra a pessoa idosa como qualquer ato, único ou repetitivo, ou omissão, que ocorra em qualquer relação supostamente de confiança, que cause danos ou incômodos, podendo ser classificada em violência física, sexual, psicológica, econômica, institucional, abandono/negligência e autonegligência (Krug, Dahlberg, Zwi \& Lozano, 2002). A dependência física e emocional, o convívio familiar e o despreparo do cuidador são fatores de risco para a prática da violência contra a pessoa idosa. $\mathrm{O}$ ato de deixar de realizar os cuidados primitivos do idoso já se caracteriza como violência sem que o praticante perceba (Oliveira et al, 2018).

A partir da década de 80 as denúncias de violências intrafamiliar contra os idosos ganharam maior visibilidade, porém, este comportamento violento é de difícil identificação, no país, atualmente os estudos que temos são insuficientes para se ter consciência da real situação. No entanto, o perfil da população brasileira atual já diz muito, como os problemas socioeconômicos, para uma grande fração da sociedade, a intolerância ao envelhecimento e a idolatria à juventude, se apresentam como elementos motivadores da disseminação da violência, fazendo crer que o problema seja bastante frequente (Aguiar et al., 2015).

Embora seja um tema pouco explorado, atualmente tem sido evidenciado devido à pandemia da COVID-19, que levou esta população ao isolamento social e com isso, ao aumento de 59\% do total das denúncias recebidas em 2019 pelo Disque 100 (de março a junho de 2020, foram 25.533 denúncias, no mesmo período de 2019, foram 16.039), tendo relevância o alerta às autoridades competentes no que diz respeito à situação da violência na saúde contra a pessoa idosa (Lüder, 2020).

As denúncias ocorrem através da Notificação que é o principal mecanismo através do qual o Ministério da Saúde (MS) recebe os dados epidemiológicos necessários para a adoção de medidas de intervenção cabíveis. A sistematização dos dados permite caracterizar os tipos e a natureza das violências cometidas, o perfil das vítimas e dos (as) prováveis autores (as) das violências (Ministério da Saúde, 2010).

Diante do descrito acima, o objetivo deste artigo foi descrever o perfil epidemiológico da violência contra a pessoa idosa de um estado da região nordeste do Brasil. Acredita-se que a identificação e a notificação da violência ajudem na elaboração de estratégias de prevenção e cuidados específicos para essa população.

\section{Metodologia}

O presente trabalho trata-se de uma pesquisa descritiva, retrospectiva, de abordagem quantitativa, na qual seguiu as normas fundamentais conforme descrito por Pereira et al. (2018). O estudo foi desenvolvido com dados do Sistema de Informação de Agravos de Notificação (SINAN) de Alagoas. A população do estudo foi constituída por 1.535 homens e mulheres com 60 anos ou mais de idade, que sofreram um os mais tipos de violência registrados e disponibilizados online pelo Departamento de Informática do Sistema Único de Saúde (DATASUS) referentes ao período de 2008 a 2018. Os dados foram 
coletados a partir da Ficha de Notificação de Violência Interpessoal/Autoprovocada que é preenchida pelas unidades assistenciais para cada paciente, quando da suspeita da ocorrência de alterações da condição de saúde de notificação compulsória ou de interesse nacional, estadual ou municipal. Esta ficha contém várias informações relacionadas à violência, mas para esse estudo, foi realizado um recorte e utilizado variáveis sexo, escolaridade, raça/cor, classificação da violência, local de ocorrência, zona de residência, unidade notificadora, tipos de violência e meio de agressão. Os dados coletados foram inseridos em um banco do software Microsoft Excel ${ }^{\circledR}$ versão 2020, analisados utilizando a estatística descritiva simples (número relativo e percentual) e apresentados na forma de gráficos e tabelas, os quais foram utilizados para a interpretação dos mesmos tomando-se como aporte a literatura referente à temática.

Este estudo utilizou dados secundários, de livre acesso e sem possibilidade de identificação dos sujeitos, por conseguinte, dispensa a aprovação do Comitê de Ética em Pesquisa, respeitando os procedimentos éticos e legais.

\section{Resultados}

Foram analisadas 1.535 notificações de casos de violência interpessoal/autoprovocada em pessoas idosas, extraídas das fichas de notificação de violência interpessoal/autoprovocada. O gráfico 1 evidencia a evolução ao longo dos anos pesquisados.

Gráfico 1: Frequência das notificações de violência contra a pessoa idosa, segundo ano da notificação, Alagoas, 2008- 2018.

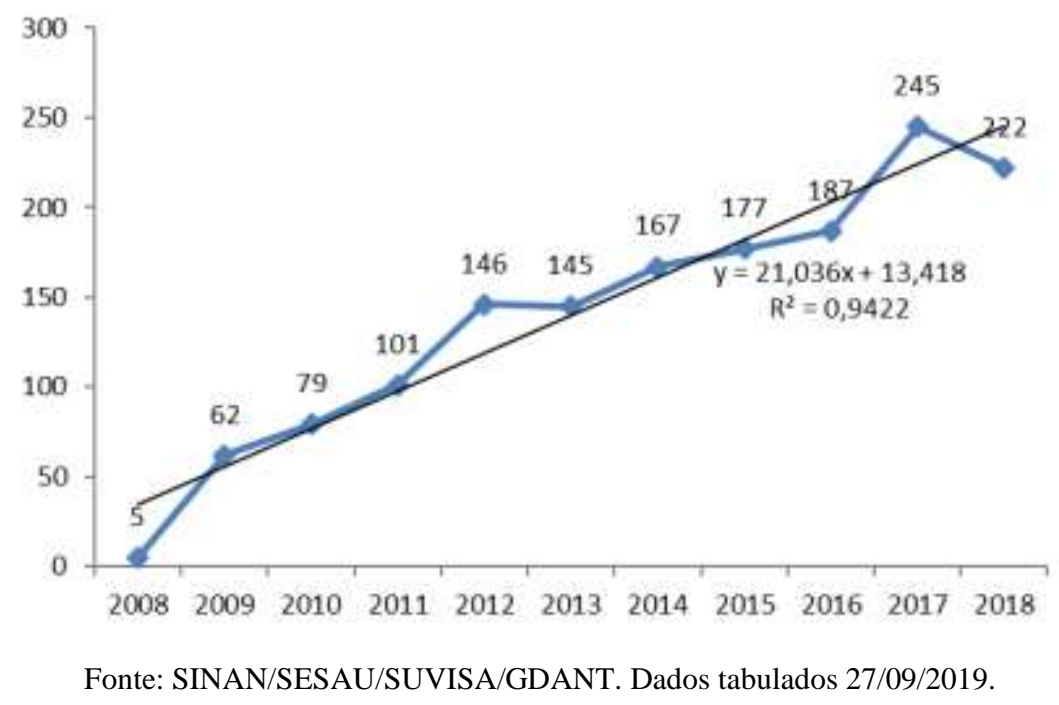

Referente às características sóciodemográficas, constatou-se que 63,4\% das pessoas idosas vítimas de violência eram homens, 34,8\% pessoas da raça parda e 11,8\% cursaram o ensino fundamental (Tabela 1). 
Research, Society and Development, v. 11, n. 2, e31611225780, 2022

(CC BY 4.0) | ISSN 2525-3409 | DOI: http://dx.doi.org/10.33448/rsd-v11i2.25780

Tabela 1: Distribuição das notificações de violência contra idosos, segundo dados sociodemográficos, Alagoas, 2008-2018.

\begin{tabular}{|c|c|c|}
\hline Variável & $\mathrm{n}^{\circ}$ & $\%$ \\
\hline \multicolumn{3}{|l|}{ Sexo } \\
\hline Masculino & 974 & 63,4 \\
\hline Feminino & 561 & 36,6 \\
\hline \multicolumn{3}{|l|}{ Escolaridade } \\
\hline Analfabetos & 185 & 12 \\
\hline Ensino fundamental & 182 & 11,8 \\
\hline Ensino médio & 34 & 2,2 \\
\hline Ensino superior & 17 & 1,1 \\
\hline Ignorados/brancos & 1117 & 72,8 \\
\hline \multicolumn{3}{|l|}{ Raça/cor } \\
\hline Parda & 534 & 34,8 \\
\hline Branca & 129 & 8,4 \\
\hline Preta & 61 & 4,0 \\
\hline Indígena & 15 & 1,0 \\
\hline Amarela & 14 & 0,9 \\
\hline Ignorados/brancos & 782 & 50,9 \\
\hline Total & 1.535 & 100 \\
\hline
\end{tabular}

* Ign= ignorados. Fonte: SINAN/SESAU/SUVISA/GDANT Dados tabulados em 27/09/2019.

Quanto às características da violência identificamos que $74,4 \%$ foram interpessoais, $37,9 \%$, ocorreram na residência da vítima, 56,8\% na zona urbana e 37,2\% foram notificadas por unidades de emergência (Tabela 2). Devido a problemas de não preenchimento adequado da ficha de notificação de violência, não foi possível identificar o vínculo do agressor. 
Tabela 2: Distribuição das notificações de violência contra idosos, segundo local de ocorrência, zona de residência e unidade notificadora, Alagoas, 2008-2018.

\begin{tabular}{|c|c|c|}
\hline Variável & $\mathrm{n}^{\mathrm{o}}$ & $\%$ \\
\hline \multicolumn{3}{|c|}{ Classificação da violência } \\
\hline Interpessoal & 1142 & 74,4 \\
\hline Autoprovocada & 247 & 16,1 \\
\hline Ign/brancos & 146 & 9,5 \\
\hline \multicolumn{3}{|l|}{ Local de ocorrência } \\
\hline Residência & 582 & 37,9 \\
\hline Via publica & 180 & 11,7 \\
\hline Outros & 68 & 4,4 \\
\hline Ign/brancos & 705 & 45,9 \\
\hline \multicolumn{3}{|l|}{ Zona de residência } \\
\hline Periurbana & 12 & 0,8 \\
\hline Urbana & 872 & 56,8 \\
\hline Rural & 426 & 27,7 \\
\hline Ign/brancos & 225 & 14,7 \\
\hline \multicolumn{3}{|c|}{ Unidade de saúde notificadora } \\
\hline Unid. De emergência & 572 & 37,2 \\
\hline Hospital geral & 488 & 31,8 \\
\hline Outras & 475 & 30,9 \\
\hline Total & 1.535 & 100 \\
\hline
\end{tabular}

* ign=ignorados. Fonte: SINAN/SESAU/SUVISA/GDANT. Dados tabulados 27/09/2019.

Destacamos que o número de dados ignorados e brancos, nesse estudo, foi muito elevado em quase todas as variáveis pesquisadas e esse resultado pode estar relacionado a ausência de algumas informações da ficha de notificação, não realizadas durante o preenchimento.

Em relação ao tipo de violência, $77 \%$ foi do tipo física sendo $82,1 \%$ no sexo masculino. O meio de agressão mais frequente, foi a força corporal/espancamento $(40,1 \%)$ sendo $42,8 \%$ no sexo feminino (Tabela 3 ). 
Tabela 3: Perfil das notificações de violência interpessoal/autoprovocada em idosos, segundo o tipo de violência e o meio de agressão, Alagoas. 2008-2018.

\begin{tabular}{|c|c|c|c|c|c|c|}
\hline \multirow{3}{*}{ Variável } & \multicolumn{4}{|l|}{ Sexo } & \multirow{3}{*}{$\begin{array}{l}\text { Total } \\
\mathrm{n}^{\circ}\end{array}$} & \multirow[b]{3}{*}{$\%$} \\
\hline & \multicolumn{2}{|c|}{ Masculino } & \multicolumn{2}{|c|}{ Feminino } & & \\
\hline & $\mathrm{n}^{\circ}$ & $\%$ & $\mathrm{n}^{\mathrm{o}}$ & $\%$ & & \\
\hline & 974 & 63,4 & 561 & 36,6 & 1.535 & 100 \\
\hline \multicolumn{7}{|l|}{ Tipo de violência } \\
\hline Física & 800 & 82,1 & 383 & 68,3 & 1.183 & 77,0 \\
\hline Psicológica & 53 & 5,4 & 98 & 17,5 & 151 & 9,8 \\
\hline Negligência/abandono & 34 & 3,4 & 75 & 13,3 & 109 & 7,2 \\
\hline Outros & 116 & 11,9 & 136 & 24,2 & 252 & 16,4 \\
\hline Total & 1003 & 102,8 & 692 & 123,3 & 1.695 & 110,4 \\
\hline \multicolumn{7}{|l|}{ Meio de agressão } \\
\hline Força corporal/espancamento & 375 & 38,5 & 240 & 42,8 & 616 & 40,1 \\
\hline Objeto perfurocortante & 170 & 17,5 & 55 & 9,8 & 225 & 14,7 \\
\hline Arma de fogo & 128 & 13,1 & 38 & 6,8 & 166 & 10,8 \\
\hline Objeto contundente & 106 & 10,9 & 35 & 6,2 & 141 & 9,2 \\
\hline Outros & 162 & 16,6 & 180 & 32,0 & 342 & 22.2 \\
\hline Total & 941 & 96,6 & 548 & 97,6 & 1.490 & 74,8 \\
\hline
\end{tabular}

Fonte: SINAN/SESAU/SUVISA/GDANT. Dados tabulados 27/09/2019.

\section{Discussão}

O presente estudo analisou a violência praticada contra a pessoa idosa, a qual é considerada de rápida propagação, e por deixar sequelas traumáticas fazem dela um problema de saúde pública, resultando em traumas físicos, morais e psicoemocionais, podendo levar a vítima a um quadro de incapacidade, dependência e até mesmo morte (Rodrigues et al., 2017). Acredita-se que fatores como a vulnerabilidade física e mental dos idosos, os transtornos emocionais do agressor e o estresse do cuidador sejam responsáveis pelo alto índice de violência contra a pessoa idosa (Miziara et al., 2015).

No período estudado foi observado um aumento significativo no número de notificações a partir do ano de 2011. Neste ano, foi alterado o artigo 19 do Estatuto do Idoso pela Lei $\mathrm{N}^{\mathrm{o}} 12.461$, onde os casos de violência contra a pessoa idosa atendida em unidades de saúde passaram a ser comunicados compulsoriamente (Ministério da Saúde, 2016). Essa alteração pode ter influenciado no aumento das notificações. Esse aumento também foi identificado no estudo quantitativo de Silva et al. (2018). Entendemos que a notificação é compulsória no âmbito da Saúde e não configura denúncia, mas sim um instrumento de garantia de direitos, dessa forma necessita ser realizada, e de forma completa.

Apesar dessa conquista, o estatuto do idoso por si só não é garantia de segurança para essa população, além de leis e políticas públicas, os idosos precisam ter consciência das agressões sofridas e denunciar esses abusos (Silva et al., 2018).

A relação da baixa escolaridade e o risco aumentado de violência na saúde da pessoa idosa é justificada pela maior necessidade, por parte do idoso, de assistência financeira, dependência da família para ajudar nas atividades de vida diária, além da dificuldade de acesso às redes de cuidado disponíveis (Irigaray, Esteves, Pacheco, Grassi-Oliveira \& Argimon, 2016). Estudos realizados em Florianopolis por Bolsoni et al., (2016) e no Rio Grande do Sul por Hohendorff et al. (2018) tiveram resultado similar. 
Na presente investigação houve predomínio de baixo nível de instrução, corroborando com achados de outros estudos (Rodrigues et al., 2017) em que os sujeitos com mais anos de educação são menos propensos a sofrerem agressões quando comparados aos que tem menor grau de instrução (Maia et al., 2019).

A respeito do predomínio da raça parda $(34,8 \%)$, dados semelhantes são descritos pelo Instituto Brasileiro de Geografia e Estatística (IBGE, 2010), onde a maioria das pessoas com 60 anos ou mais se autodeclaram pardos (66,89\%), seguido de brancos $(29,44 \%)$ e negros $(9,53 \%)$ (IBGE, 2010). É possível encontrar resultado similar em outras partes do País, como na região Centro Oeste (Soares \& Barbosa, 2020) e no estado da Paraíba (Souza, 2017). Por outro lado, o resultado aqui apresentado contraria a investigação realizada no município de São Paulo (Machado, Kimura, Duarte \& Lebrão, 2020) parte integrante do estudo SABE (Saúde, Bem-estar e Envelhecimento), com 1.126 idosos, que encontrou uma prevalência de 59,1\% para a raça branca.

Em relação à classificação da violência, neste estudo a interpessoal é prevalente $(16,1 \%)$ corroborando com outros estudos realizados em outras localidades do País como em Recife (Paraíba \& Silva 2015), Paraíba (Souza, 2017) e Caruaru-PE (Lopes et al., 2018) mas a autoprovocada também é citada por Soares e Barbosa (2020) com um percentual de 6,4\%. Podemos inferir a importância de uma eficiente rede de apoio, com enfoque interdisciplinar e multidisciplinar, agregando profissionais qualificados para reconhecer e conduzir esses casos de violência da melhor forma.

O local de ocorrência da violência predominante foi na residência da vítima com 37,9\% do total das notificações, em outras pesquisas (Paraíba \& Silva, 2015, Irigaray et al., 2016, Meleiro et al., 2021) a residência da vítima também foi identificada como principal local de escolha para prática da violência. O estudo de Lino et al. (2019), traz que a dependência do idoso como fator causador de sobrecarga ao cuidador aumentando o risco de violência na própria residência da vítima, diz também que essa agressão é praticada de forma sutil, o que a faz ser confundida com estresse interpessoal.

Ainda sobre o assunto, Silva e Dias (2016) trazem que na maioria das vezes o agressor faz uso abusivo de álcool, depende financeiramente ou mora na casa do idoso, tem laços fracos com a vítima por ter sofrido violência/abuso ou até mesmo ter sido abandonado na infância, fazendo destes fatores contribuintes para o aumento do risco do idoso sofrer violência em sua residência.

De acordo com Oliveira (2018) por diversos motivos como vergonha, medo de ser abandonado, sofrer punição, ser institucionalizado, o idoso tende a negar e justificar a violência sofrida para proteger membros da família ou seu cuidador, sendo assim, a violência intrafamiliar ainda é de difícil identificação e segue velada e naturalizada por quem a sofre resultando em alto índice de subnotificações.

Diante da observação dos dados acerca da zona de residência mais frequente, a zona urbana foi predominante com 56,8\% dos casos notificados, corroborando, esse resultado, com outros estudos (Hohendorff et al., 2018, Soares \& Barbosa, 2020, Lopes et al., 2018).

Referente à Unidade notificadora, a presente pesquisa revelou que a maioria dos casos de violência, 69\%, foi notificada nas unidades de emergência. Andrade et al. (2020), no estudo que teve como objetivo caracterizar os casos de violência notificados no Sistema de Informação de Agravos de Notificação (SINAN) da 8 a Regional de Saúde, afirmam que ao sofrer violência, caso a vítima precise de atendimento na rede de saúde em consequência da agressão, os profissionais que prestarem atendimento, devem realizar a notificação.

Quando comparado o percentual de violência sexual com outros tipos de violência, neste estudo encontramos que em 1,9\% dos casos notificados ocorreu violência sexual, resultado similar foi constatado em outros estudos (Bolsoni et al., 2016, Hohendorff et al., 2018). 
Em Alagoas, através da Portaria da Secretaria de Saúde do Estado (SESAU) nº 1.900, de 29 de maio de 2018, a violência autoprovocada e a sexual passaram a ser de notificação imediata para o Estado. Foi um marco importante para dar visibilidade a essa violência que sempre existiu, mas muitas vezes não era conhecida (Irigaray et al., 2016).

Dantas et al. (2018), mostram que o baixo número de notificações a respeito da violência sexual se dá à invisibilidade deste tipo de violência, devido à dificuldade por parte dos profissionais de saúde na identificação e notificação dos casos ocorridos, causando escassez na divulgação de dados reais.

Acredita-se que grande parte dos casos de violência sexual cometidos contra o idoso não são notificados por vários motivos, tais como constrangimento, medo ou sentimento de culpa em denunciar o agressor, especialmente se fizer parte da família, quanto pelo fato de a maioria dos casos ser praticado por um familiar, geralmente filho ou filha. O estudo realizado por Souza et al. (2021) também identificou esse resultado. É importante destacar que nem sempre a violência sexual resultará em internação hospitalar, onde geralmente ocorre à notificação, sendo assim muitos casos estão subnotificados (Machado et al., 2020).

A violência física toma destaque no estudo, representando 71,9\% dos casos, com maior expressividade no sexo masculino (52,1\%). Este resultado também é encontrado em outros estudos (Paraíba \& Silva, 2015, Hohendorff et al., 2018), os quais trazem que a pessoa idosa sofre diversas formas de agressão simultaneamente, porém nem sempre são de fácil identificação seja pela falta de denúncia por parte das vítimas seja pela não revelação do caso por parte dos agressores. Provavelmente isso explicaria o fato da violência física ter sido mais expressiva, pois é mais facilmente detectada.

Este estudo traz a força corporal/espancamento como o principal meio de agressão, representando $40 \%$ dos casos, outros estudos (Hohendorff et al., 2018, Soares \& Barbosa, 2020) realizados Rio Grande do Sul e no Planalto Central, respectivamente, corroboram com este achado.

\section{Conclusão}

A prevalência da violência no período descrito ocorreu na própria residência de pessoas idosas do sexo masculino, da raça parda, residentes na zona urbana, que eram alfabetizadas, vítimas de violência física ocorrida através da força corporal/espancamento e a maior parte dessas notificações foram feitas por unidades de emergência.

Dentre as limitações da investigação tivemos a incompletude das fichas de notificação que podem ocorrer por desconhecimento dos profissionais acerca do processo de notificação, por mudanças ocorridas na rotina do serviço de saúde fazendo com que ocorra uma descontinuidade na notificação, dentre outras. Outra limitação se refere à escassez de estudos a respeito do tema, principalmente em relação ao Estado pesquisado.

Essas limitações não diminuem a importância do estudo, pois o mesmo traz visibilidade ao tema da violência contra a pessoa idosa, no entanto, as fichas devem ser preenchidas corretamente pois os resultados são de extrema importância para a elaboração de políticas públicas específicas.

\section{Referências}

Aguiar, M. P. C., Leite, H. A., Dias, I. M., Mattos, M. C. T. \& Lima, W. R. (2015). Violência contra idosos: descrição de casos no Município de Aracaju, Sergipe, Brasil. Revista de Enfermagem Escola Anna Nery, 9(2), 343-349.

Andrade, C. M., Teixeira, G. T., França, T. B., Rambo, M., Trevvisan, M. G., Casaril, E. \& Costa, L. D. (2020) Violência interpessoal e autoprovocada: caracterização dos casos notificados em uma regional de saúde do paraná. Rev Cogitare Enfermagem, v25i0.63758.

Bolsoni, C. C, Coelho, E. B. S, Giehl, M. W. C \& d’Orsi, E. (2016). Prevalência de violência contra idosos e fatores associados, estudo de base populacional em Florianópolis, SC. Revista Brasileira de Geriatria e Gerontologia. 19 (4), 671-682.

Brasil. (2010). Ministério da Saúde (MS). Secretaria de Atenção à Saúde. Departamento de Ações Programáticas Estratégicas. Linha de Cuidado para a Atenção Integral à Saúde de Crianças, Adolescentes e suas Famílias em Situação de Violência Orientações para gestores e profissionais de saúde. Brasília: MS. 
Dantas, N. M. F, Silva, F. C., Oliveira, R. R. \& Batista, J. L. F. P. (2018). Internações de idosas vítimas de agressão sexual no Brasil. Revista Interdisciplinar em Violência e Saúde, 1.

Hohendorff, J. V., Paz, A. P., Freitas, C. P. P., Lawrenz, P., \& Habigzang, L. F. (2018). Caracterização da violência contra idosos a partir de casos notificados por profissionais da saúde: Revista da SPAGESP. 19 (2), 64-80.

Souza, C. S. (2017). Violência contra os idosos: perfil epidemiológico das notificações no estado da Paraíba (Trabalho de Conclusão de Curso). Universidade Federal de Campina Grande, Campina Grande, Paraíba.

Instituto Brasileiro de Geografia e Estatística. (2010). População residente, por raça ou cor, segundo a situação do domicílio, o sexo e a idade. IBGE

Irigaray, T. Q., Esteves, C. F., Pacheco, J. T. B., Grassi-Oliveira, R. \& Argimon, J. I. L. (2016). Maus-tratos contra idosos em Porto Alegre, Rio Grande do Sul: um estudo documental. Revista Psicologia da Saúde. 33 (3), 543-55.

Krug, E. G., Dahlberg, L. L., Mercy, J. A., Zwi, A. B. \& Lozano, R. (Eds.). (2002). Relatório mundial sobre violência e saúde. OMS.

Lino, V. T. S., Rodrigues, N. C. P., Lima, I. S., Athie, S., \& Souza, E. R., (2019). Prevalência e fatores associados ao abuso de cuidadores contra idosos dependentes: a face oculta da violência familiar. Revista Ciência \& Saúde Coletiva. 24(1), 87-96.

Lopes, L. G. F., Leal, M. C. C., Souza, E. F., Silva, S. Z. R., Guimarães, N. N. A., \& Silva, L. S. R. (2018). Violência contra a pessoa idosa. Revista Enfermagem. UFPE on line. 12(9),2257-2268.

Lüder A. Cresce 59\% o número de denúncias de violência contra o idoso no Brasil durante a pandemia da Covid-19. Globo News. 2020 Oct 29.

Machado, D. R., Kimura, M., Duarte, Y. A. O., \& Lebrão, M. L., (2020). Violência contra idosos e qualidade de vida relacionada à saúde: estudo populacional no município de São Paulo, Brasil. Revista Ciência \& Saúde Coletiva. 25(3),1119-1128.

Maia, P. H. S., Ferreira, E. F., Melo, E. M. \& Vargas, A. M. D. (2019). A ocorrência da violência em idosos e seus fatores associados. Revista Brasileira de Enfermagem. 72(2),71-7.

Meleiro, M. L. A. P., Nascimento, I. R., Santos, F. S., Silva, N. P. M \& Nascimento V. (2021). A violência contra a pessoa idosa em Manaus e no Amazonas/Brasil. Research, Society and Development, 10(5), e11210514558.

Ministério da Saúde. (2016) Secretaria de Vigilância em Saúde. Departamento de Vigilância de Doenças e Agravos Não Transmissíveis e Promoção da Saúde.Viva: instrutivo de notificação de violência doméstica, sexual e outras violências. (2a ed.), DF. 94 p.

Miziara C. S. M. G., Braga, M. V., Carvalho, F. I., Teixeira, T. V., Miziara, I. D. \& Muñoz, D. R. (2015). Vítima silenciosa: violência doméstica contra o idoso no Brasil. Revista Saúde Ética \& Justiça. 20(1),1-8.

Oliveira, K. S. M., Carvalho, F. P. B., Oliveira, L. C., Simpson, C. A., Silva, F. T. L. \& Mart, A. G. C. (2018). Violência contra idosos: concepções dos profissionais de enfermagem acerca da detecção e prevenção. Revista Gaúcha de Enfermagem.39.

Paraíba, P. M. F \& Silva M. C. M. (2015). Perfil da violência contra a pessoa idosa na cidade do Recife-PE. Revista Brasileira de Geriatria e Gerontologia. $18(2), 295-306$

Pereira, A. S., Shitisuka, D. M., Parreira, F. J. \& Shitisuka, R. (2018). Metodologia da pesquisa científica. UFSM.

Rogrigues, R. A. P., Monteiro, E. A., Santos, A. M. R., Pontes, L. M. F., Fhon, J. R. S, Bolina, A. F., Seredynskyj, F. L., Almeida, V. C., Giacomini, S. B. L., Defina, G. P. C. \& Silva, L. M. (2017). Violência contra idosos em três municípios brasileiros. Rev Bras Enferm. 70(4).

Silva, C. F. S \& Dias C. M. S. B (2016). Violência Contra Idosos na Família: Motivações, Sentimentos e Necessidades do Agressor. Revista Psicologia: Ciência e Profissão. 36 (3), 637-652.

Silva, G. C. N., Almeida, V. L., Brito, T. R. P., Godinho, M. L. C., Nogueira, D. A. \& Chini, L. T. (2018) Violência contra idosos: uma análise documental. Revista Aquichan.18(4), 449-460.

Soares, M. C \& Barbosa, A. M. (2020). Perfil de idosos vítimas de violência atendidos em um hospital de urgências. Revista Científica da Escola Estadual de Saúde Pública de Goiás "Cândido Santiago". 6(1):18-34.

Souza, E. A. B., Silva, B. C., Cabral, L. P., Filho, N. J. S., Zimmermann, I. M. M. \& Zimmermann, R. D. Violência contra idosos relatada em notícias durante a pandemia do novo coronavírus. Research, Society and Development, 10(14). e57101420046. 